\title{
Anisotropic SD2 brane: accelerating cosmology and Kasner-like space-time from compactification
}

\author{
Kuntal Nayek $^{1,2, a}$, Shibaji Roy ${ }^{1,2, b}$ \\ ${ }^{1}$ Saha Institute of Nuclear Physics, 1/AF, Bidhannagar, Calcutta 700064, India \\ ${ }^{2}$ Homi Bhabha National Institute, Training School Complex, Anushakti Nagar, Mumbai 400085, India
}

Received: 5 May 2017 / Accepted: 27 June 2017 / Published online: 12 July 2017

(C) The Author(s) 2017. This article is an open access publication

\begin{abstract}
Starting from an anisotropic (in all directions including the time direction of the brane) non-SUSY D2 brane solution of type IIA string theory we construct an anisotropic space-like D2 brane (or SD2 brane, for short) solution by the standard trick of a double Wick rotation. This solution is characterized by five independent parameters. We show that compactification on six-dimensional hyperbolic space $\left(\mathrm{H}_{6}\right)$ of a time-dependent volume of this SD2 brane solution leads to accelerating cosmologies (for some time $t \sim t_{0}$, with $t_{0}$ some characteristic time) where both the expansions and the accelerations are different in three spatial directions of the resultant four-dimensional universe. On the other hand at early times $\left(t \ll t_{0}\right)$ this four-dimensional space, in certain situations, leads to four-dimensional Kasnerlike cosmology, with two additional scalars, namely, the dilaton and a volume scalar of $\mathrm{H}_{6}$. Unlike in the standard fourdimensional Kasner cosmology here all three Kasner exponents could be positive definite, leading to expansions in all three directions.
\end{abstract}

\section{Introduction}

It is well known [1] that a cosmological solution of a higherdimensional vacuum Einstein equation can give rise to interesting four-dimensional cosmology (with a period of accelerated expansion) upon time-dependent hyperbolic space compactifications [2-4]. This process, therefore, evades a no-go theorem $[5,6]$ of obtaining such accelerated expansion in standard time-independent compactifications. Similar cosmologies also follow if one includes fluxes [7] and/or a dilaton field [8] in the higher-dimensional theories such as $\mathrm{M} /$ string theory. $\mathrm{M} /$ string theory solution which gives rise to four-dimensional accelerating cosmologies upon time-

\footnotetext{
a e-mail: kuntal.nayek@saha.ac.in

be-mail: shibaji.roy@saha.ac.in
}

dependent hyperbolic space compactifications is called the space-like M2 (SM2) brane (for M theory) or space-like D2 (SD2) brane (for string theory). Space-like branes $[9,10]$ are topological defects localized on a space-like hypersurface and exist for a moment in time. So, they are time-dependent solutions of field theories or $\mathrm{M} / \mathrm{string}$ theory with an isometry $\mathrm{ISO}(p+1) \times \mathrm{SO}(d-p-2,1)$ for an $\mathrm{S} p$ brane in $d$ space-time dimensions [11-14]. The original motivation for constructing these solutions was to understand the time-dependent processes in field and M/string theory $[9,10,15,16]$ and also to have a better understanding of the dS/CFT correspondence $[17,18]$. The cosmological implication leading to fourdimensional accelerated expansion from these solutions has been elucidated in Refs. [1,7,8,19-24].

The previous $\mathrm{S} 2$ brane solutions considered in the literature $[7,8,11-14]$ were isotropic in the brane directions and so the four-dimensional accelerating cosmologies obtained from these solutions were isotropic. In this paper we will construct an anisotropic SD2 brane solutions of type IIA string theory and try to see whether similar four-dimensional accelerating cosmologies can be obtained in all three spatial directions upon compactification. Another motivation to look at the anisotropic SD2 brane solution is to see whether one can get a four-dimensional Kasner-like [25] solution from it upon compactification where one can get expansions in all three spatial directions which is not possible in conventional Kasner solution from four-dimensional vacuum Einstein equation. The construction of anisotropic SD2 brane solution follows from the standard double Wick rotation [26] of the known anisotropic non-SUSY D2 brane solution [27] of type IIA string theory. This solution is characterized by five independent parameters. We then cast the solution in a suitable time-like coordinate and is given in terms of a single harmonic function containing a characteristic time $t_{0}$. Next, we compactify the space-time on a six-dimensional hyperbolic space with time-dependent volume. The resultant metric when expressed in an Einstein frame gives us a four- 
dimensional FLRW type space-time with three different scale factors in three spatial directions. We find that when $t \sim t_{0}$, we can get accelerating cosmologies in all three directions when other parameters of the solution take some specific values. Although the expansions and the accelerations in all three directions are not the same but they do not differ drastically and the accelerations are all transient. However, when $t \ll t_{0}$, the resultant four-dimensional metric takes a Kasnerlike form when the parameters characterizing the solution satisfy certain conditions. But because of the presence of the dilaton as well as the volume scalar of the six-dimensional hyperbolic space, all the Kasner exponents could be positive definite, leading to expansions in all three spatial directions. However, the expansions in this case are decelerating. This can be contrasted with the standard four-dimensional Kasner space-time [25] (obtained from the solution of the vacuum Einstein equation) where expansions in all three directions are not possible.

This paper is organized as follows. In the next section we give the construction of anisotropic SD2 brane solution from its time-like counterpart and cast the solution in a coordinate system suitable for our purpose. In Sect. 3, we obtain the anisotropic accelerating cosmologies from this solution upon compactification on six-dimensional hyperbolic space of a time-dependent volume. In Sect. 4, we show how a fourdimensional Kasner-like geometry arises from this string theory solution, where all the Kasner exponents could be positive definite leading to expansions in all three spatial directions unlike the standard Kasner solution in four dimensions. Finally, we conclude in Sect. 5.

\section{Anisotropic SD2 brane solutions}

In this section we construct the anisotropic SD2 brane solution from the known anisotropic non-SUSY D2 brane solution of type IIA string theory. In [27], we have constructed an anisotropic non-SUSY D $p$ brane solution and showed how it nicely interpolates between a black D $p$ brane and a KaluzaKlein "bubble of nothing" when some of the parameters of the solution are varied continuously and interpreted this interpolation as closed string tachyon condensation. Here we make use of that solution and write the anisotropic non-SUSY D2 brane solution in the following by putting $p=2$ in Eq. (4) (we have replaced $\delta_{0}$ by $\delta_{3}$ for convenience) of Ref. [27],

$$
\begin{aligned}
\mathrm{d} s^{2}= & F(r)^{\frac{3}{8}}(H(r) \tilde{H}(r))^{\frac{2}{5}}\left(\frac{H(r)}{\tilde{H}(r)}\right)^{\frac{\delta_{1}}{4}+\frac{\delta_{2}}{10}+\frac{\delta_{3}}{10}} \\
& \times\left(\mathrm{d} r^{2}+r^{2} \mathrm{~d} \Omega_{6}^{2}\right) \\
& +F(r)^{-\frac{5}{8}}\left\{-\left(\frac{H(r)}{\tilde{H}(r)}\right)^{\frac{\delta_{1}}{4}+\frac{\delta_{2}}{2}+\frac{\delta_{3}}{2}} \mathrm{~d} t^{2}\right.
\end{aligned}
$$

$$
\begin{aligned}
+ & \left(\frac{H(r)}{\tilde{H}(r)}\right)^{-\frac{3 \delta_{1}}{4}-\frac{3 \delta_{2}}{2}+\frac{\delta_{3}}{2}}\left(\mathrm{~d} x^{1}\right)^{2} \\
& \left.+\left(\frac{H(r)}{\tilde{H}(r)}\right)^{-\frac{3 \delta_{1}}{4}+\frac{\delta_{2}}{2}-\frac{3 \delta_{3}}{2}}\left(\mathrm{~d} x^{2}\right)^{2}\right\} \\
e^{2\left(\phi-\phi_{0}\right)}= & F(r)^{\frac{1}{2}}\left(\frac{H(r)}{\tilde{H}(r)}\right)^{\delta_{1}-2 \delta_{2}-2 \delta_{3}}, \quad F_{[6]}=\hat{Q} \operatorname{Vol}\left(\Omega_{6}\right)
\end{aligned}
$$

The metric in the above is given in the Einstein frame. The various functions appearing in the solution are defined as

$$
\begin{aligned}
& F(r)=\left(\frac{H(r)}{\tilde{H}(r)}\right)^{\alpha} \cosh ^{2} \theta-\left(\frac{\tilde{H}(r)}{H(r)}\right)^{\beta} \sinh ^{2} \theta, \\
& H(r)=1+\frac{\omega^{5}}{r^{5}}, \quad \tilde{H}(r)=1-\frac{\omega^{5}}{r^{5}} .
\end{aligned}
$$

Note that the solution has eight parameters $\alpha, \beta, \delta_{1}, \delta_{2}, \delta_{3}$, $\theta, \omega$, and $\hat{Q} . \phi_{0}$ is the asymptotic value of the dilaton, $F_{[6]}$ is a six form and $\hat{Q}$ is the magnetic charge associated with the D2 brane. The solution becomes isotropic in the brane directions when $\delta_{1}=-2 \delta_{2}=-2 \delta_{3}$. So, in that sense these parameters can be called anisotropy parameters. Now for the consistency of the field equations the eight parameters of the solution must satisfy the following relations [27]:

$$
\begin{aligned}
& \alpha-\beta=-\frac{3}{2} \delta_{1} \\
& \frac{1}{2} \delta_{1}^{2}+\frac{1}{2} \alpha\left(\alpha+\frac{3}{2} \delta_{1}\right)+\frac{2}{5} \delta_{2} \delta_{3}=\frac{6}{5}\left(1-\delta_{2}^{2}-\delta_{3}^{2}\right) \\
& \hat{Q}=5 \omega^{5}(\alpha+\beta) \sinh 2 \theta .
\end{aligned}
$$

These three relations reduce the number of independent parameters from eight to five, which are $\omega, \theta$, and the anisotropy parameters $\delta_{1}, \delta_{2}$ and $\delta_{3}$. Using the second and the first relations in (4), we can express $\alpha$ and $\beta$ in terms of the other parameters as

$$
\begin{aligned}
& \alpha=-\frac{3}{4} \delta_{1} \pm \frac{1}{2} \sqrt{\frac{48}{5}\left(1-\delta_{2}^{2}-\delta_{3}^{2}\right)-\frac{7}{4} \delta_{1}^{2}-\frac{16}{5} \delta_{2} \delta_{3}} \\
& \beta=\frac{3}{4} \delta_{1} \pm \frac{1}{2} \sqrt{\frac{48}{5}\left(1-\delta_{2}^{2}-\delta_{3}^{2}\right)-\frac{7}{4} \delta_{1}^{2}-\frac{16}{5} \delta_{2} \delta_{3}}
\end{aligned}
$$

The form of the harmonic function $\tilde{H}(r)$ in (3) indicates that there is a naked singularity of the solution at $r=\omega$ and therefore, the solution is well defined only for $r>\omega$. Now we apply the double Wick rotation [26] $r \rightarrow i \tau, t \rightarrow-i x^{3}$ to the solution (1) along with $\omega \rightarrow i \omega, \theta \rightarrow i \theta$ and $\theta_{1} \rightarrow i \theta_{1}$, where $\theta_{1}$ is one of the angular coordinates of the sphere $\Omega_{6}$ of the transverse space. This operation gives us anisotropic space-like D2 brane from the anisotropic static non-SUSY D2 brane and the change in the angular coordinate converts spherical $\Omega_{6}$ to hyperbolic $H_{6}$. Thus the transformed solution is, 


$$
\begin{aligned}
\mathrm{d} s^{2}= & F(\tau)^{\frac{3}{8}}(H(\tau) \tilde{H}(\tau))^{\frac{2}{5}} \\
& \times\left(\frac{H(\tau)}{\tilde{H}(\tau)}\right)^{\frac{\delta_{1}}{4}+\frac{\delta_{2}}{10}+\frac{\delta_{3}}{10}}\left(-\mathrm{d} \tau^{2}+\tau^{2} \mathrm{~d} H_{6}^{2}\right) \\
& +F(\tau)^{-\frac{5}{8}}\left\{\left(\frac{H(\tau)}{\tilde{H}(\tau)}\right)^{\frac{\delta_{1}}{4}+\frac{\delta_{2}}{2}+\frac{\delta_{3}}{2}}\left(\mathrm{~d} x^{3}\right)^{2}\right. \\
& +\left(\frac{H(\tau)}{\tilde{H}(\tau)}\right)^{-\frac{3 \delta_{1}}{4}-\frac{3 \delta_{2}}{2}+\frac{\delta_{3}}{2}}\left(\mathrm{~d} x^{1}\right)^{2} \\
& \left.+\left(\frac{H(\tau)}{\tilde{H}(\tau)}\right)^{-\frac{3 \delta_{1}}{4}+\frac{\delta_{2}}{2}-\frac{3 \delta_{3}}{2}}\left(\mathrm{~d} x^{2}\right)^{2}\right\}, \\
e^{2\left(\phi-\phi_{0}\right)}= & F(\tau)^{\frac{1}{2}}\left(\frac{H(\tau)}{\tilde{H}(\tau)}\right)^{\delta_{1}-2 \delta_{2}-2 \delta_{3}}, \\
F_{[6]}= & \hat{Q} \operatorname{Vol}\left(H_{6}\right) .
\end{aligned}
$$

The various functions associated with the solution are also changed under the above rotation and are given below,

$$
\begin{aligned}
& F(\tau)=\left(\frac{H(\tau)}{\tilde{H}(\tau)}\right)^{\alpha} \cos ^{2} \theta+\left(\frac{\tilde{H}(\tau)}{H(\tau)}\right)^{\beta} \sin ^{2} \theta, \\
& H(\tau)=1+\frac{\omega^{5}}{\tau^{5}}, \quad \tilde{H}(\tau)=1-\frac{\omega^{5}}{\tau^{5}} .
\end{aligned}
$$

Thus we see that the anisotropic static non-SUSY D2 brane has been converted to anisotropic time-dependent or spacelike D2 brane. For the former solution the radial coordinate $r$ was transverse to the D2 brane's world-volume, whereas, for the latter the time-like coordinate $\tau$ is transverse to the SD2 brane's world-volume. The metric of the transverse sphere $\mathrm{d} \Omega_{6}^{2}$ has been converted to negative of the metric of the hyperbolic space $\mathrm{d} H_{6}^{2}$. The hyperbolic functions $\sinh ^{2} \theta$ and $\cosh ^{2} \theta$ become $-\sin ^{2} \theta$ and $\cos ^{2} \theta$ respectively, therefore, the relative sign of the two terms of the function $F(\tau)$ has been flipped. But the form field remains unchanged with $\hat{Q} \rightarrow-\hat{Q}$. Thus the first two parameter relations in (4) remain the same, while the last relation has changed to $\hat{Q}=5 \omega^{5}(\alpha+\beta) \sin 2 \theta$. Now for our purpose we will make a coordinate transformation from $\tau$ to $t$ given by

$\tau=t\left(\frac{1+\sqrt{g(t)}}{2}\right)^{\frac{2}{5}}$, where $g(t)=1+\frac{4 \omega^{5}}{t^{5}} \equiv 1+\frac{t_{0}^{5}}{t^{5}}$

Under this coordinate change we have

$$
\begin{aligned}
& H(\tau)=1+\frac{\omega^{5}}{\tau^{5}}=\frac{2 \sqrt{g(t)}}{1+\sqrt{g(t)}}, \\
& \tilde{H}(\tau)=1-\frac{\omega^{5}}{\tau^{5}}=\frac{2}{1+\sqrt{g(t)}},
\end{aligned}
$$

$$
\begin{gathered}
H(\tau) \tilde{H}(\tau)=\frac{4 \sqrt{g(t)}}{(1+\sqrt{g(t)})^{2}}, \quad \frac{H(\tau)}{\tilde{H}(\tau)}=\sqrt{g(t)}, \\
-\mathrm{d} \tau^{2}+\tau^{2} \mathrm{~d} H_{6}^{2}=g(t)^{\frac{1}{5}}\left(-\frac{\mathrm{d} t^{2}}{g(t)}+t^{2} \mathrm{~d} H_{6}^{2}\right) .
\end{gathered}
$$

Using (10) we can rewrite the anisotropic SD2 brane solution given in (6) as follows:

$$
\begin{aligned}
& \mathrm{d} s^{2}=F(t)^{\frac{3}{8}} g(t)^{\frac{\delta_{1}}{8}+\frac{\delta_{2}}{20}+\frac{\delta_{3}}{20}+\frac{1}{5}}\left(-\frac{\mathrm{d} t^{2}}{g(t)}+t^{2} \mathrm{~d} H_{6}^{2}\right) \\
& +F(t)^{-\frac{5}{8}}\left[g(t)^{-\frac{3 \delta_{1}}{8}-\frac{3 \delta_{2}}{4}+\frac{\delta_{3}}{4}}\left(\mathrm{~d} x^{1}\right)^{2}\right. \\
& \left.+g(t)^{-\frac{3 \delta_{1}}{8}+\frac{\delta_{2}}{4}-\frac{3 \delta_{3}}{4}}\left(\mathrm{~d} x^{2}\right)^{2}+g(t)^{\frac{\delta_{1}}{8}+\frac{\delta_{2}}{4}+\frac{\delta_{3}}{4}}\left(\mathrm{~d} x^{3}\right)^{2}\right], \\
& e^{2\left(\phi-\phi_{0}\right)}=F(t)^{\frac{1}{2}} g(t)^{\frac{\delta_{1}}{2}-\delta_{2}-\delta_{3}}, \quad F_{[6]}=\hat{Q} \operatorname{Vol}\left(H_{6}\right)
\end{aligned}
$$

where $g(t)$ is as given in (9) and $F(t)$ is given by

$F(t)=g(t)^{\frac{\alpha}{2}} \cos ^{2} \theta+g(t)^{-\frac{\beta}{2}} \sin ^{2} \theta$.

It is important to note that in the new coordinate, the original singularity at $\tau=\omega$ has been shifted to $t=0$. Also note that as $t \gg t_{0}, g(t), F(t) \rightarrow 1$ and therefore, the solution reduces to flat space. In the next two sections we will impose the assumption $t \sim t_{0}$ and also $t \ll t_{0}$ into the solution (11) to see how one can get accelerating cosmology in the first case and a Kasner-like cosmology in the second case in $(3+1)$ dimensions upon compactification.

\section{Compactification and accelerating cosmology}

In this section we will compactify the anisotropic SD2 brane solution given in (11) on a six-dimensional hyperbolic space of a time-dependent volume and write the resultant four-dimensional metric in the Einstein frame. ${ }^{1}$ This fourdimensional metric will have the standard FLRW form whose cosmology we want to study. We rewrite the metric in (11) in a four-dimensional part and the transverse six-dimensional part as

$\mathrm{d} s^{2}=\mathrm{d} s_{4}^{2}+e^{2 \psi} \mathrm{d} H_{6}^{2}$

\footnotetext{
${ }^{1}$ Here one might ask: since hyperbolic spaces are in general noncompact in what sense are we compactifying the ten-dimensional space on six-dimensional hyperbolic space and studying the four-dimensional cosmology? To address this question we remark that it is quite well known how to construct compact hyperbolic manifolds (CHMs) from hyperbolic spaces and there is a vast mathematical literature some of which are given in [2-4]. In short, the CHMs are obtained from $H_{d}$ (with $\mathrm{d} \geq 2$ ), the universal covering space of $d$-dimensional hyperbolic manifold by modding out by an appropriate freely acting discrete subgroup of the isometry group $\mathrm{SO}(1, d)$ of $H_{d}$. CHMs have many interesting properties and we refer the reader to some of the original literature [2-4] for details.
} 
where $\psi$ is the radion field and $e^{2 \psi}=F(t)^{\frac{3}{8}}$ $g(t)^{\frac{\delta_{1}}{8}+\frac{\delta_{2}}{20}+\frac{\delta_{3}}{20}+\frac{1}{5}} t^{2}$. The four-dimensional metric $\mathrm{d} s_{4}^{2}$ is given as

$$
\begin{aligned}
\mathrm{d} s_{4}^{2}= & -F(t)^{\frac{3}{8}} g(t)^{\frac{\delta_{1}}{8}+\frac{\delta_{2}}{20}+\frac{\delta_{3}}{20}-\frac{4}{5}} \mathrm{~d} t^{2}+F(t)^{-\frac{5}{8}} \\
& \times\left[g(t)^{-\frac{3 \delta_{1}}{8}-\frac{3 \delta_{2}}{4}+\frac{\delta_{3}}{4}}\left(\mathrm{~d} x^{1}\right)^{2}\right. \\
& \left.+g(t)^{-\frac{3 \delta_{1}}{8}+\frac{\delta_{2}}{4}-\frac{3 \delta_{3}}{4}}\left(\mathrm{~d} x^{2}\right)^{2}+g(t)^{\frac{\delta_{1}}{8}+\frac{\delta_{2}}{4}+\frac{\delta_{3}}{4}}\left(\mathrm{~d} x^{3}\right)^{2}\right]
\end{aligned}
$$

The compactified four-dimensional metric (14) when expressed in an Einstein frame takes the form [8],

$$
\begin{aligned}
\mathrm{d} s_{4 E}^{2}= & e^{6 \psi} \mathrm{d} s_{4}^{2} \\
= & -F(t)^{\frac{3}{2}} g(t)^{-\frac{1}{5}+\frac{\delta_{1}}{2}+\frac{\delta_{2}}{5}+\frac{\delta_{3}}{5}} t^{6} \mathrm{~d} t^{2} \\
& +F(t)^{\frac{1}{2}} g(t)^{\frac{3}{5}-\frac{3 \delta_{2}}{5}+\frac{2 \delta_{3}}{5}} t^{6} \mathrm{~d} x_{1}^{2} \\
& +F(t)^{\frac{1}{2}} g(t)^{\frac{3}{5}+\frac{2 \delta_{2}}{5}-\frac{3 \delta_{3}}{5}} t^{6} \mathrm{~d} x_{2}^{2} \\
& +F(t)^{\frac{1}{2}} g(t)^{\frac{3}{5}+\frac{\delta_{1}}{2}+\frac{2 \delta_{2}}{5}+\frac{2 \delta_{3}}{5}} t^{6} \mathrm{~d} x_{3}^{2} \\
= & -A(t)^{2} \mathrm{~d} t^{2}+\sum_{i=1}^{3} S_{i}(t)^{2} \mathrm{~d} x_{i}^{2}
\end{aligned}
$$

where the various time-dependent coefficients are

$$
\begin{aligned}
& A(t)=F(t)^{\frac{3}{4}} g(t)^{-\frac{1}{10}+\frac{\delta_{1}}{4}+\frac{\delta_{2}}{10}+\frac{\delta_{3}}{10} t^{3},} \\
& S_{1}(t)=F(t)^{\frac{1}{4}} g(t)^{\frac{3}{10}-\frac{3 \delta_{2}}{10}+\frac{\delta_{3}}{5}} t^{3}, \\
& S_{2}(t)=F(t)^{\frac{1}{4}} g(t)^{\frac{3}{10}+\frac{\delta_{2}}{5}-\frac{3 \delta_{3}}{10} t^{3},} \\
& S_{3}(t)=F(t)^{\frac{1}{4}} g(t)^{\frac{3}{10}+\frac{\delta_{1}}{4}+\frac{\delta_{2}}{5}+\frac{\delta_{3}}{5} t^{3} .}
\end{aligned}
$$

Note that in the compactified four-dimensional space there are three fields, namely $g_{\mu \nu}, \phi, \psi$. Now we perform another coordinate transformation,

$$
\begin{aligned}
\mathrm{d} \eta^{2} & =F(t)^{\frac{3}{2}} g(t)^{-\frac{1}{5}+\frac{\delta_{1}}{2}+\frac{\delta_{2}}{5}+\frac{\delta_{3}}{5}} t^{6} \mathrm{~d} t^{2} \Rightarrow, \\
\eta & =\int F(t)^{\frac{3}{4}} g(t)^{-\frac{1}{10}+\frac{\delta_{1}}{4}+\frac{\delta_{2}}{10}+\frac{\delta_{3}}{10} t^{3} \mathrm{~d} t},
\end{aligned}
$$

and rewrite the Einstein frame metric $\mathrm{d} s_{4 E}^{2}$ in the standard flat FLRW form as

$\mathrm{d} s_{4 E}^{2}=-\mathrm{d} \eta^{2}+s_{i}^{2}(\eta) \sum_{i=1}^{3}\left(\mathrm{~d} x^{i}\right)^{2}$

with $\eta$ being the canonical time and the scale factor $s_{i}(\eta) \equiv$ $S_{i}(t)$. Note that since $s_{i}(\eta)$ are different for each $i$, the cosmology here will be anisotropic. Now because of the complicated relation between $t$ and $\eta$ let us define (see, for example $[23,24])$

$$
\begin{aligned}
& m_{i}(t) \equiv \frac{\mathrm{d} \ln S_{i}(t)}{\mathrm{d} t} \\
& n_{i}(t) \equiv\left[\frac{\mathrm{d}^{2}}{\mathrm{~d} t^{2}} \ln \left(S_{i}(t)\right)+\frac{\mathrm{d}}{\mathrm{d} t} \ln \left(S_{i}(t)\right) \frac{\mathrm{d}}{\mathrm{d} t} \ln \left(\frac{S_{i}(t)}{A(t)}\right)\right]
\end{aligned}
$$

and with these one can easily see that $m_{i}(t)>0$ implies that $\mathrm{d} s_{i}(\eta) / \mathrm{d} \eta>0$, amounting to expansion of our universe, and similarly, $n_{i}(t)>0$ implies that $\mathrm{d}^{2} s_{i}(\eta) / \mathrm{d} \eta^{2}>0$, amounting to acceleration of our universe. Therefore, from (19) it is clear that in the four-dimensional space-time (15) we get an accelerated expansion in the $i$ th coordinate direction only if the parameters $m_{i}(t)$ and $n_{i}(t)$ are simultaneously positive in that direction. It can be checked that, for $t \ll t_{0}$, accelerating expansion is not possible at all in any direction. However, it is possible only if $t \sim t_{0}$. In this case the first term in the harmonic function $g(t)$ given in (9) is of the same order as the second. The other parameters of the solution, namely, $\delta_{1}, \delta_{2}$ and $\delta_{3}$ cannot be totally arbitrary. From Eq. (5), we see that the reality of $\alpha$ and $\beta$ imposes some restriction on the value of these three anisotropy parameters. Also it can be checked that by changing the value of $\theta$ does not change the cosmological behavior of the solution very much. Thus we have chosen some typical values of these parameters (as given in the figure) and plotted the functions $m_{i}(t)$ and $n_{i}(t)$ in Fig. 1, to show that it is indeed possible to have accelerating expansions in all three directions.

We notice as shown in (a)-(c) in Fig. 1, we always get expanding universe (given by the solid blue line) in all three directions, but the expansion is accelerating only for a short period of time, i.e., the acceleration is transient (given by the dotted red line). Also note that since $m_{i}(t)$ and $n_{i}(t)$ are different for different $i$, the cosmology is anisotropic, however, the anisotropy is not too much.

To understand the accelerating expansion, we can write down the four-dimensional compactified action from the original ten-dimensional one and obtain the form of the potential of the dilaton and the radion field [8]. The tendimensional action has the form,

$S=\int \mathrm{d}^{10} x \sqrt{-g}\left[R-\frac{1}{2}(\partial \phi)^{2}-\frac{1}{2 \cdot 6 !} e^{-\phi / 2} F_{[6]}^{2}\right]$.

Reducing the action on a six-dimensional hyperbolic space $H_{6}$, the four-dimensional action we get $^{2}[20,23,24,28]$

$S_{4}=\int \mathrm{d}^{4} x \sqrt{-g_{4 E}}\left[R_{4 E}-\frac{1}{2}(\partial \phi)^{2}-24(\partial \psi)^{2}-V(\phi, \psi)\right]$

${ }^{2}$ Here reduction on the hyperbolic space $H_{6}$ to obtain the fourdimensional action is done in the sense decribed in footnote 3 . This has also been done in the references $[20,28]$. 


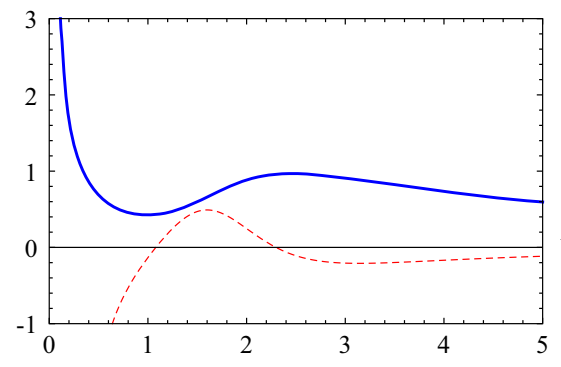

(a) along $x_{1}$ direction

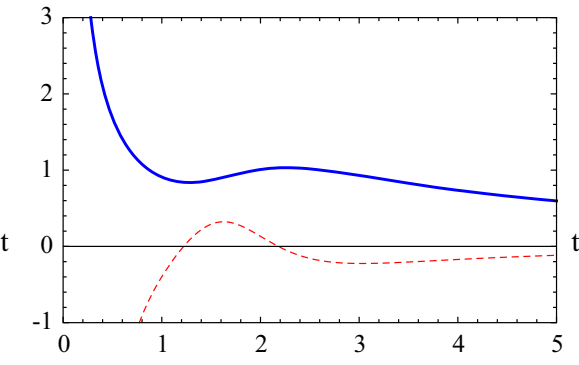

(b) along $x_{2}$ direction

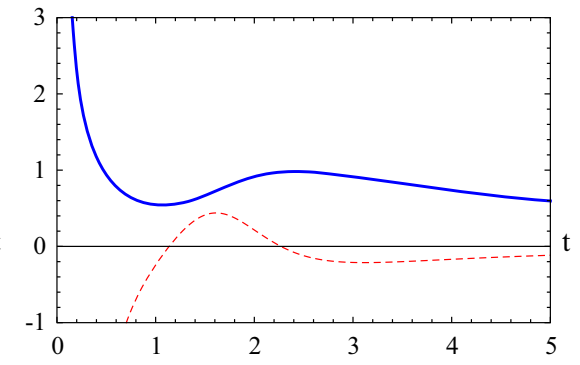

(c) along $x_{3}$ direction

Fig. 1 The plot of $m(t)$ (solid blue line) and $n(t)$ (dashed red line) in different spatial coordinate directions at $\theta=\pi / 6, \delta_{1}=-0.5, \delta_{2}=0.2, \delta_{3}=$ 0.4 and $t_{0}=2.0$

where

$V(\phi, \psi)=\frac{\hat{Q}^{2}}{2} e^{-\frac{\phi}{2}-18 \psi}+30 e^{-8 \psi}$.

Here $\hat{Q}$ is the magnetic charge of the D2 brane given in (1). Note that because of the hyperbolic space compactification the potential is always positive irrespective of the charge and therefore there is always a possibility that the system will be driven to an accelerating phase (see the [20]).

\section{Compactification and Kasner-like solution}

In this section we will show how a four-dimensional Kasnerlike cosmological solution follows from the anisotropic SD2 brane solution upon the six-dimensional hyperbolic space compactification discussed in the previous section. The compactified action expressed in Einstein frame is given in (15). We take this four-dimensional metric and express it at early times, $t \ll t_{0}$. In this case the function $g(t)$ can be approximated by

$g(t)=1+\frac{t_{0}^{5}}{t^{5}} \approx \frac{t_{0}^{5}}{t^{5}} \sim t^{-5}$.

Also since we want to express the metric components in (15) as some powers of $t$, we note from the form of $F(t)$ in (12) that this can be done (assuming $\alpha>0$ without any loss of generality) in three ways as follows. (a) Put $\theta=0$, with $\alpha, \beta$ as given in (5), (b) put $\alpha=-\beta=-(3 / 4) \delta_{1}$, with $\theta$ arbitrary and (c) both $\alpha>0, \beta>0$, with $\theta$ arbitrary. There is another possibility with $\theta=\pi / 2$ and $\beta<0$, but this case can be seen to be equivalent to case (a). Note that for case (a) and (b) we have $\hat{Q}=0$ ( since $\left.\hat{Q}=5 \omega^{5}(\alpha+\beta) \sin 2 \theta\right)$, however, for case (c) $\hat{Q}$ is non-zero and the non-SUSY brane is magnetically charged. In either case (a) or (b) we have

$$
F(t) \sim t^{-\frac{5 \alpha}{2}}
$$

In the above we have absorbed $t_{0}$ in $t$. But for case (c) $F(t)$ has an additional $\cos ^{2} \theta$ factor which can be absorbed in $t$ as well as in $x^{1,2,3}$. Thus in all cases $F(t)$ has the form as given in (24). So, in this near region, the space-time metric (15), the dilaton and the radion fields take the forms,

$$
\begin{aligned}
& \mathrm{d} s^{2}=-t^{2\left(\frac{7}{2}-\frac{15 \alpha}{8}-\frac{5 \delta_{1}}{4}-\frac{\delta_{2}}{2}-\frac{\delta_{3}}{2}\right)} \mathrm{d} t^{2}+t^{2\left(\frac{3}{2}-\frac{5 \alpha}{8}+\frac{3 \delta_{2}}{2}-\delta_{3}\right)}\left(\mathrm{d} x^{1}\right)^{2} \\
& +t^{2\left(\frac{3}{2}-\frac{5 \alpha}{8}-\delta_{2}+\frac{3 \delta_{3}}{2}\right)}\left(\mathrm{d} x^{2}\right)^{2}+t^{2\left(\frac{3}{2}-\frac{5 \alpha}{8}-\frac{5 \delta_{1}}{4}-\delta_{2}-\delta_{3}\right)}\left(\mathrm{d} x^{3}\right)^{2} \\
& e^{2\left(\phi-\phi_{0}\right)}=t^{2\left(-\frac{5 \alpha}{8}-\frac{5 \delta_{1}}{4}+\frac{5 \delta_{2}}{2}+\frac{5 \delta_{3}}{2}\right)} \\
& e^{2 \psi}=t^{2\left(\frac{1}{2}-\frac{15}{32} \alpha-\frac{5}{16} \delta_{1}-\frac{\delta_{2}}{8}-\frac{\delta_{3}}{8}\right)} .
\end{aligned}
$$

Now since we are taking $t \ll 1$ here, we have to be careful about the validity of the gravity solution. The gravity solution will be valid as long as the dilaton remains small and the curvature of the transverse space in string units also remains small. These two conditions impose certain restrictions on the parameters of the solution and they are given as

$$
\begin{aligned}
& 5 \alpha+5 \delta_{1}-4 \delta_{2}-4 \delta_{3}>4 \\
& \alpha+2 \delta_{1}-4 \delta_{2}-4 \delta_{3} \leq 0
\end{aligned}
$$

where $\alpha$ is as given in (5). Furthermore, the reality of $\alpha$ also restricts the parameters as

$\frac{35}{4} \delta_{1}^{2}+48 \delta_{2}^{2}+48 \delta_{3}^{2}+16 \delta_{2} \delta_{3} \leq 48$

We have checked numerically that all these three conditions can be satisfied simultaneously for a certain range of values of the parameters $\delta_{1}, \delta_{2}$ and $\delta_{3}$ and only for those values we have a valid gravity solution (25). We would like to remark here that the validity of the supergravity solution also requires that we cannot take $t$ arbitrarily close to zero as we are considering $t \ll 1$. In fact $t$ has to be much larger than the string scale if the supergravity solution remains valid. This can be seen if we calculate $\dot{\phi}^{2}, \dot{\psi}^{2}$ and also the scalar curvature with the solution given in (25). All of these terms come out to be proportional to $1 / t^{2}$ and so, when $t \ll 1$, they can become very large invalidating the supergravity solution 
and stringy corrections must be included. To avoid this we require $\sqrt{\alpha^{\prime}} \ll t \ll t_{0}$ or in terms of scaled $t$, we must have $\sqrt{\alpha^{\prime}} / t_{0} \ll t \ll 1$. Now, keeping those restrictions in mind, we can rewrite the solution in terms of canonical time

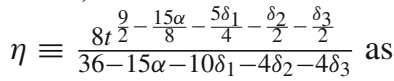

$\mathrm{d} s^{2}=-\mathrm{d} \eta^{2}+\eta^{2 p_{1}}\left(\mathrm{~d} x^{1}\right)^{2}+\eta^{2 p_{2}}\left(\mathrm{~d} x^{2}\right)^{2}+\eta^{2 p_{3}}\left(\mathrm{~d} x^{3}\right)^{2}$, $e^{2\left(\phi-\phi_{0}\right)}=C\left(\delta_{1}, \delta_{2}, \delta_{3}\right) \eta^{2 \gamma_{\phi}} \quad e^{2 \psi}=D\left(\delta_{1}, \delta_{2}, \delta_{3}\right) \eta^{2 \gamma_{\psi}}$.

Note that in writing the metric in (28) we have rescaled the coordinates $x^{1}, x^{2}$ and $x^{3}$ by some constant factors involving the parameters $\delta_{1}, \delta_{2}, \delta_{3}$. Also in the dilaton and the radion field $C$ and $D$ are constants involving these paramaters whose explicit form will not be important. It can be easily checked in the defining relation of $\eta$ that the coefficient in front of $t$ is always positive definite and that also ensures that as $t \rightarrow 0$, $\eta \rightarrow 0$. The Kasner exponents $p_{1}, p_{2}$ and $p_{3}$ in the metric and $\gamma_{\phi}, \gamma_{\psi}$ are defined as

$$
\begin{aligned}
p_{1} & =\frac{12-5 \alpha+12 \delta_{2}-8 \delta_{3}}{36-15 \alpha-10 \delta_{1}-4 \delta_{2}-4 \delta_{3}} \\
p_{2} & =\frac{12-5 \alpha-8 \delta_{2}+12 \delta_{3}}{36-15 \alpha-10 \delta_{1}-4 \delta_{2}-4 \delta_{3}} \\
p_{3} & =\frac{12-5 \alpha-10 \delta_{1}-8 \delta_{2}-8 \delta_{3}}{36-15 \alpha-10 \delta_{1}-4 \delta_{2}-4 \delta_{3}} \\
\gamma_{\phi} & =\frac{-5 \alpha-10 \delta_{1}+20 \delta_{2}+20 \delta_{3}}{36-15 \alpha-10 \delta_{1}-4 \delta_{2}-4 \delta_{3}} \\
\gamma_{\psi} & =\frac{1}{4} \frac{16-15 \alpha-10 \delta_{1}-4 \delta_{2}-4 \delta_{3}}{36-15 \alpha-10 \delta_{1}-4 \delta_{2}-4 \delta_{3}} .
\end{aligned}
$$

Now since this a solution to the compactified four-dimensional action given in (21), it must satisfy the equations of motion. The Einstein equation, the dilaton and radion equations following from (21) have the forms

$$
\begin{aligned}
& R_{\mu \nu, E}-\frac{1}{2} \partial_{\mu} \phi \partial_{\nu} \phi-24 \partial_{\mu} \psi \partial_{\nu} \psi=0 \\
& \frac{1}{\sqrt{-g_{E}}} \partial_{\mu}\left(\sqrt{-g_{E}} g_{E}^{\mu \nu} \partial_{\nu} \phi\right)=0, \\
& \frac{1}{\sqrt{-g_{E}}} \partial_{\mu}\left(\sqrt{-g_{E}} g_{E}^{\mu \nu} \partial_{\nu} \psi\right)=0 ;
\end{aligned}
$$

here $\mu, v$ run over $(1+3)$-dimensional space-time. Note that since we have $t \ll 1$, the potential in (22) is trivial (the first term is zero even when $\hat{Q} \neq 0$ because the exponential factor effectively goes to zero due to the relations given in (26) and similarly the exponential in the second term also effectively goes to zero because of (26)). Substituting the above solution (28) in (30), we get two conditions

$$
p_{1}+p_{2}+p_{3}=1, \quad \text { and } \quad p_{1}^{2}+p_{2}^{2}+p_{3}^{2}=1-\frac{1}{2} \gamma_{\phi}^{2}-24 \gamma_{\psi}^{2} \text {. }
$$

The first condition of (31) can be seen to be satisfied trivially from (29). On the other hand when we substitute the parameter values from (29) to the second condition of (31), we find that it gives the same parametric relation as the second relation of (4) verifying the consistency of the solution. This therefore shows how one can get a four-dimensional Kasnerlike solution from the ten-dimensional anisotropic SD2 brane solution by six-dimensional hyperbolic space compactification. It is well known that the standard Kasner solution [25], obtained as the solution of the vacuum Einstein equation, does not lead to expansions in all spatial directions. The reason is that in standard Kasner cosmology the Kasner exponents satisfy $p_{1}+p_{2}+p_{3}=1$ and $p_{1}^{2}+p_{2}^{2}+p_{3}^{2}=1$. Since these two conditions cannot be satisfied together when the $p_{i}$ are all positive, the expansions cannot occur in all the directions. However, for the four-dimensional Kasner cosmology we obtained from string theory solutions, the parameters $p_{i}$ can all be positive definite because the second condition here (31) is different. This is essentially the reason that we can have expansions in all the directions, but it can easily be checked that the expansions are decelerating.

\section{Conclusion}

To summarize, in this paper we have constructed an anisotropic SD2 brane solution starting from an anisotropic nonSUSY D2 brane solution of type IIA string theory by the standard trick of a double Wick rotation. We wanted to see whether it is possible to generate accelerating cosmologies in all the directions which is known for the isotropic SD2 brane solution upon compactification on the six-dimensional hyperbolic space of a time-dependent volume. Indeed we found that when the resultant four-dimensional metric is expressed in Einstein frame there are some windows of the parameters of the solution where one can get accelerating cosmologies in all the directions and is discussed in Sect. 3. Here both the expansions and the accelerations we found are anisotropic. But in order to get accelerating expansions we noted that the anisotropy cannot be too drastic in three different directions. We also noted that accelerations are possible only for $t \sim t_{0}$, where $t_{0}$ is some characteristic time given as one of the parameters of the solution. Next, we looked at the four-dimensional metric at early times, i.e., for $t \ll t_{0}$ and found that in a suitable coordinate and under certain conditions on the parameters of the solution, it can be expressed in a standard four-dimensional Kasner-like form. But unlike in the standard Kasner cosmology, where expansions in all three directions are not possible, here we can get expansions in all the three directions. The reason is that in this case the relations among the Kasner exponents get modified due to the presence of the dilaton and the radion field. It would be interesting to see what effect (such modification to Kasner 
solution at early time) it would have on the cosmological singularities [29-31].

Open Access This article is distributed under the terms of the Creative Commons Attribution 4.0 International License (http://creativecomm ons.org/licenses/by/4.0/), which permits unrestricted use, distribution, and reproduction in any medium, provided you give appropriate credit to the original author(s) and the source, provide a link to the Creative Commons license, and indicate if changes were made.

Funded by SCOAP ${ }^{3}$.

\section{References}

1. P.K. Townsend, M.N.R. Wohlfarth, Accelerating cosmologies from compactification. Phys. Rev. Lett. 91, 061302 (2003). arXiv:hep-th/0303097

2. N. Kaloper, J. March-Russell, G.D. Starkman, M. Trodden, Compact hyperbolic extra dimensions: Branes, Kaluza-Klein modes and cosmology. Phys. Rev. Lett. 85, 928 (2000). arXiv:hep-ph/0002001

3. G.D. Starkman, D. Stojkovic, M. Trodden, Large extra dimensions and cosmological problems. Phys. Rev. D 63, 103511 (2001). arXiv:hep-th/0012226

4. G.D. Starkman, D. Stojkovic, M. Trodden, Homogeneity, flatness and 'large' extra dimensions. Phys. Rev. Lett. 87, 231303 (2001). arXiv:hep-th/0106143

5. G.W. Gibbons, Aspects of supergravity theories, in Supersymmetry, Supergravity and Related Topics, ed. by F. de Aguila, J.A. de Azcarraga, L. Ibanez (World Scientific, Singapore, 1985), p. 346

6. J.M. Maldacena, C. Nunez, Supergravity description of field theories on curved manifolds and a no go theorem. Int. J. Mod. Phys. A 16, 822 (2001). arXiv:hep-th/0007018

7. N. Ohta, Accelerating cosmologies from S-branes. Phys. Rev. Lett. 91, 061303 (2003). arXiv:hep-th/0303238

8. S. Roy, Accelerating cosmologies from M/string theory compactifications. Phys. Lett. B 567, 322 (2003). arXiv:hep-th/0304084

9. M. Gutperle, A. Strominger, Space-like branes. JHEP 0204, 018 (2002). arXiv:hep-th/0202210

10. A. Maloney, A. Strominger, X. Yin, S-brane thermodynamics. JHEP 0310, 048 (2003). arXiv:hep-th/0302146

11. C.-M. Chen, D.V. Gal'tsov, M. Gutperle, S brane solutions in supergravity theories. Phys. Rev. D 66, 024043 (2002). arXiv:hep-th/0204071

12. M. Kruczenski, R.C. Myers, A.W. Peet, Supergravity S-branes. JHEP 0205, 039 (2002). arXiv:hep-th/0204144
13. S. Roy, On supergravity solutions of space-like Dp-branes. JHEP 0208, 025 (2002). arXiv:hep-th/0205198

14. S. Bhattacharya, S. Roy, Time dependent supergravity solutions in arbitrary dimensions. JHEP 0312, 015 (2003). arXiv:hep-th/0309202

15. A. Sen, NonBPS states and branes in string theory. arXiv:hep-th/9904207

16. A. Sen, Rolling tachyon. JHEP 0204, 048 (2002). arXiv:hep-th/0203211

17. A. Strominger, The dS/CFT correspondence. JHEP 0110, 034 (2001). arXiv:hep-th/0106113

18. M. Spradlin, A. Strominger, A. Volovich, Les Houches lectures on de Sitter space. arXiv:hep-th/0110007

19. N. Ohta, A study of accelerating cosmologies from superstring/M theories. Prog. Theor. Phys. 110, 269 (2003). arXiv:hep-th/0304172

20. R. Emparan, J. Garriga, A note on accelerating cosmologies from compactifications and S branes. JHEP 0305, 028 (2003). arXiv:hep-th/0304124

21. C.-M. Chen, P.-M. Ho, I.P. Neupane, N. Ohta, J.E. Wang, Hyperbolic space cosmologies. JHEP 0310, 058 (2003). arXiv:hep-th/0306291

22. S.B. Giddings, R.C. Myers, Spontaneous decompactification. Phys. Rev. D 70, 046005 (2004). arXiv:hep-th/0404220

23. S. Roy, H. Singh, Space-like branes, accelerating cosmologies and the near 'horizon' limit. JHEP 0608, 024 (2006). arXiv:hep-th/0606041

24. K. Nayek, S. Roy, Space-like D $p$ branes: accelerating cosmologies versus conformally de Sitter space-time. JHEP 1502, 021 (2015). arXiv:1411.2444 [hep-th]

25. E. Kasner, Geometrical theorems on Einstein's cosmological equations. Am. J. Math. 43, 217 (1921)

26. J.X. Lu, S. Roy, Static, non-SUSY p-branes in diverse dimensions. JHEP 0502, 001 (2005). arXiv:hep-th/0408242

27. J.X. Lu, S. Roy, Z.L. Wang, R.J. Wu, Intersecting non-SUSY branes and closed string tachyon condensation. Nucl. Phys. B 813, 259 (2009). arXiv:0710.5233 [hep-th]

28. J. Garriga, A. Vilenkin, Solutions to the cosmological constant problems. Phys. Rev. D 64, 023517 (2001). doi:10.1103/ PhysRevD.64.023517. arXiv:hep-th/0011262

29. N. Engelhardt, T. Hertog, G.T. Horowitz, Holographic signatures of cosmological singularities. Phys. Rev. Lett. 113, 121602 (2014). arXiv:1404.2309 [hep-th]

30. N. Engelhardt, T. Hertog, G.T. Horowitz, Further holographic investigations of big bang singularities. JHEP 1507, 044 (2015). arXiv:1503.08838 [hep-th]

31. S. Chatterjee, S.P. Chowdhury, S. Mukherji, Y.K. Srivastava, Nonvacuum AdS cosmology and comments on gauge theory correlator. Phys. Rev. D 95(4), 046011 (2017). arXiv:1608.08401 [hep-th] 